\title{
Article
}

\section{Novel in vitro dynamic metabolic system for predicting the human pharmacokinetics of tolbutamide}

\author{
Cai-fu XUE ${ }^{1}$, Zhe ZHANG ${ }^{1}$, Yan $J_{I N}{ }^{1}$, Bin ZHU ${ }^{2}$, Jun-fen XING ${ }^{2}$, Guo MA ${ }^{1}$, Xiao-qiang XIANG ${ }^{1}$, Wei-min $C A{ }^{1,}$ * \\ ${ }^{1}$ Department of Clinical Pharmacy, School of Pharmacy, Fudan University, Shanghai 201203, China; ${ }^{2}$ Shanghai BaiO Technology \\ Company, Shanghai 200233, China
}

\begin{abstract}
Liver metabolism is commonly considered the major determinant in drug discovery and development. Many in vitro drug metabolic studies have been developed and applied to understand biotransformation. However, these methods have disadvantages, resulting in inconsistencies between in vivo and in vitro experiments. A major factor is that they are static systems that do not consider the transport process in the liver. Here we developed an in vitro dynamic metabolic system (Bio-PK metabolic system) to mimic the human pharmacokinetics of tolbutamide. Human liver microsomes (HLMs) encapsulated in a F127'-Acr-Bis hydrogel (FAB hydrogel) were placed in the incubation system. A microdialysis sampling technique was used to monitor the metabolic behavior of tolbutamide in hydrogels. The measured results in the system were used to fit the in vitro intrinsic clearance of tolbutamide with a mathematical model. Then, a PBPK model that integrated the corresponding in vitro intrinsic clearance was developed to verify the system. Compared to the traditional incubation method, reasonable PK profiles and the in vivo clearance of tolbutamide could be predicted by integrating the intrinsic clearance of tolbutamide obtained from the Bio-PK metabolic system into the PBPK model. The predicted maximum concentration $\left(C_{\max }\right)$, area under the concentration-time curve (AUC), time to reach the maximum plasma concentration ( $\left.T_{\max }\right)$ and in vivo clearance were consistent with the clinically observed data. This novel in vitro dynamic metabolic system can compensate for some limitations of traditional incubation methods; it may provide a new method for screening compounds and predicting pharmacokinetics in the early stages, supporting the development of compounds.
\end{abstract}

Keywords: physiologically based pharmacokinetic model; FAB hydrogel; microdialysis; tolbutamide; Bio-PK metabolic system; dynamic; human liver microsomes

Acta Pharmacologica Sinica (2018) 39: 1522-1532; doi: 10.1038/aps.2017.201; published online 12 Apr 2018

\section{Introduction}

In vitro studies of drugs are usually prioritized over in vivo ones, especially in humans. In the early stages of drug discovery and development, in vitro data can be used not only to rapidly screen compounds but also to understand the mechanisms and guide the further study of drugs and decisionmaking $^{[1,2]}$.

The liver plays an important role in the human body, involving the metabolism and elimination of drugs ${ }^{[3,4]}$. Metabolites may have more activity or toxicity than the parent drug, which impacts drug efficacy and safety in humans ${ }^{[5-7]}$. Thus, biotransformation in the liver is a determinant in the overall disposition of drugs. A reliable in vitro metabolic system

\footnotetext{
*To whom correspondence should be addressed.

E-mail weimincai@fudan.edu.cn

Received 2017-08-24 Accepted 2017-12-09
}

to predict metabolic behavior in the human body is highly desired.

Currently, many in vitro metabolic systems have been developed to explain hepatic extraction. Kinetic parameters, such as $V_{\max }$ and $K_{\mathrm{m}}$, can be determined in vitro using hepatocytes, microsomes, cytoplasm, the S-9 fraction or liver slices extracted from the liver of humans and other laboratory animals ${ }^{[8-11]}$. The intrinsic clearance $\left(\mathrm{CL}_{\mathrm{int}}=V_{\max } / K_{\mathrm{m}}\right.$ under linear conditions) obtained from these models can be converted to in vivo intrinsic clearance using various scaling methods, based on the amount of enzyme present in vivo ${ }^{[12]}$. However, these systems have several limitations, such as a scarce and inconsistent metabolic rate. As the gold standard, hepatocytes are prone to inactivation over time in culture. Moreover, available primary human hepatocytes are fewer in number and scarce, as they are mainly derived from poor-quality or poorly transplanted substances ${ }^{[13-15]}$. The incubation time for microsomes 
and recombinases is often short, which limits the generation of sufficient metabolic turnover and affects prediction of the clearance rate ${ }^{[16-18]}$. More importantly, the systems described above are static, simplifying the circulation processes occurring in the liver ${ }^{[19]}$. To better understand the metabolic behavior, a new in vitro metabolic system must be developed.

In our previous study, a hydrogel carrier (F127-acrylamidebisacrylamide, FAB hydrogel) with a polymer thermosensitive material was invented. FAB hydrogel is a reverse-phase hydrogel, with the appropriate proportion of hydrogel presenting a loose, porous three-dimensional structure suitable for liver microsomal loading. It has been confirmed that microsomes encapsulated in hydrogel still have good metabolic activity ${ }^{[20]}$. Compared to ordinary microsomes, microsomal hydrogels can be used for long-term incubation reactions due to their ability to maintain longer metabolic activity and metabolic turnover ${ }^{[20]}$. We have further developed an in vitro dynamic metabolic cycle system (Bio-PK system) to better mimic and understand the metabolic behavior. Rat liver microsomes encapsulated in a FAB hydrogel were combined with the Bio-PK system, which successfully predicted the in vivo metabolism of tolbutamide ${ }^{[21]}$. A dynamic bio-pharmacokinetic/pharmacodynamic (PK/PD) system was established and assessed to predict the PK parameters and PD effects of the model drug cyclophosphamide ${ }^{[22]}$. However, drug research in humans is the ultimate goal of drug development. Due to differences in species, it is desirable to estimate clearance in humans. Based on the above results, it is important to further apply the Bio-PK system combined with microsomal hydrogel to predict human metabolism.

The objectives of the present work are 1) to prepare a microsomal hydrogel that can encapsulate human liver microsomes; 2) to construct the Bio-PK system to obtain the in vitro intrinsic clearance of tolbutamide; 3) to build the PBPK model via integration with the in vitro intrinsic clearance obtained from the Bio-PK system to predict the clinically pharmacokinetics of tolbutamide; and, ultimately, 4) to evaluate the potential and reliability of this system in predicting human metabolic behavior and to support the further development of the system.

\section{Materials and methods}

\section{Chemicals and reagents}

Pluronic F-127, tolbutamide, and 4-hydroxytolbutamide were purchased from Sigma Aldrich (St Louis, MO, USA). The BCA Protein Assay Kit, 30\% Acr-Bis (29:1), ammonium persulfate (APS), and $\mathrm{N}, \mathrm{N}, \mathrm{N}^{\prime}, \mathrm{N}^{\prime}$-tetramethylethylenediamine (TEMED) were obtained from the Beyotime Institute of Biotechnology (Nanjing, China). Phenacetin was acquired from Aladdin (Shanghai, China). Mixed gender pooled human liver microsomes (prepared from at least 20 donors) were obtained from Corning Life Sciences (Tewksbury, MA, USA), and $\beta$-Nicotinamide adenine dinucleotide 2 -phosphate (NADPH) was acquired from Roche (Mannheim, Germany).

HPLC-grade methanol was purchased from TEDIA Company, Inc (Fairfield, USA). Water was purified using a Milli$\mathrm{Q}$ water system (Bedford, MA, USA). The distilled water was used for the extraction and preparation of samples. Other reagents and chemicals were of analytical grade.

Ringer's solution $\left(145 \mathrm{mmol} / \mathrm{L} \mathrm{Na}^{+}, 1.2 \mathrm{mmol} / \mathrm{L} \mathrm{Ca}^{2+}, 4\right.$ $\mathrm{mmol} / \mathrm{L} \mathrm{K}^{+}$, and $0.1 \mathrm{mmol} / \mathrm{L}$ ascorbic acid, $\mathrm{pH} 7.0$ ) and phosphate buffer saline (PBS) solution (137 mmol/L NaCl, 2.7 $\mathrm{mmol} / \mathrm{L} \mathrm{KCl}, 10 \mathrm{mmol} / \mathrm{L}$ sodium phosphate dibasic, and 2 $\mathrm{mmol} / \mathrm{L}$ potassium phosphate monobasic, $\mathrm{pH}$ 7.4) were prepared in our laboratory.

\section{Synthesis of F-127' and preparation of HLM-encapsulated FAB hydrogel}

The synthesis of F-127' and the FAB hydrogel preparation were as previously described ${ }^{[20]}$. Briefly, F-127', a triblock copolymer, poly (ethylene oxide)-poly (propylene oxide)-poly (ethylene oxide) (PEO99-PPO65-PEO99), was derived at both ends of the F127 polymer using acryloyl chloride to generate double bonds. The FAB hydrogel system was prepared using Acr-Bis solution, F127', APS, TEMED and PBS.

The hydrogel still had a thermo-sensitive property such that the polymer chain presented a diastolic state at $4{ }^{\circ} \mathrm{C}$ and a systolic state at $37^{\circ} \mathrm{C}$. The prepared FAB hydrogel was immersed in an HLM solution for $48 \mathrm{~h}$ at $4{ }^{\circ} \mathrm{C}$ before metabolic tests.

\section{Liquid chromatography-tandem mass spectrometry method}

Liquid chromatography-tandem mass spectrometry (LCMS/MS) analysis was conducted using an Agilent 1200 series HPLC system (Agilent Technologies, Waldbronn, Germany) coupled to an API 4000 Q Trap mass spectrometer (AB Sciex, Darmstadt, Germany) equipped with an API electrospray ionization (ESI) source.

The chromatography separation was performed on an Agilent Eclipse XDB-C18 column $(150 \mathrm{~mm} \times 2.1 \mathrm{~mm}$ id, $5 \mu \mathrm{m}$; Agilent Technologies, Waldbronn, Germany). The column temperature was maintained at $30{ }^{\circ} \mathrm{C}$. The mobile phase consisted of (A) methanol $+0.2 \%$ acetic acid and (B) water $+0.2 \%$ acetic acid by an isocratic elution of $60 \% \mathrm{~A}$ at $0-5 \mathrm{~min}$. The flow rate was $0.3 \mathrm{~mL} / \mathrm{min}$, and the injection volume was $5 \mu \mathrm{L}$.

The ESI source was operated in negative ionization mode with an ion spray voltage at $4.5 \mathrm{kV}$. The source temperature was set to $500{ }^{\circ} \mathrm{C}$. Multiple reaction monitoring (MRM) was performed for each sample. The ion transitions were mass-tocharge ratios of $(\mathrm{m} / \mathrm{z}) 269.1$ to 169.8 for tolbutamide, 285.0 to 185.8 for 4-hydroxytolbutamide and 178.1 to 148.8 for phenacetin, which was used as the internal standard. All data were analyzed using Analyst software, version 1.6.

\section{Construction of the Bio-PK metabolic system}

The Bio-PK metabolic system consisted of a peristaltic pump and an incubation system, tightly connected by a recirculation pipeline. HLMs encapsulated in the FAB hydrogel were placed in the incubation system. Substrate and NADPH were also added to the incubation system. The final incubation system was placed in a thermostatic metal bath. A microdialysis sampling technique was used to obtain the free concentration levels of drugs in hydrogels. A schematic of the Bio-PK system is shown in Figure 1. 


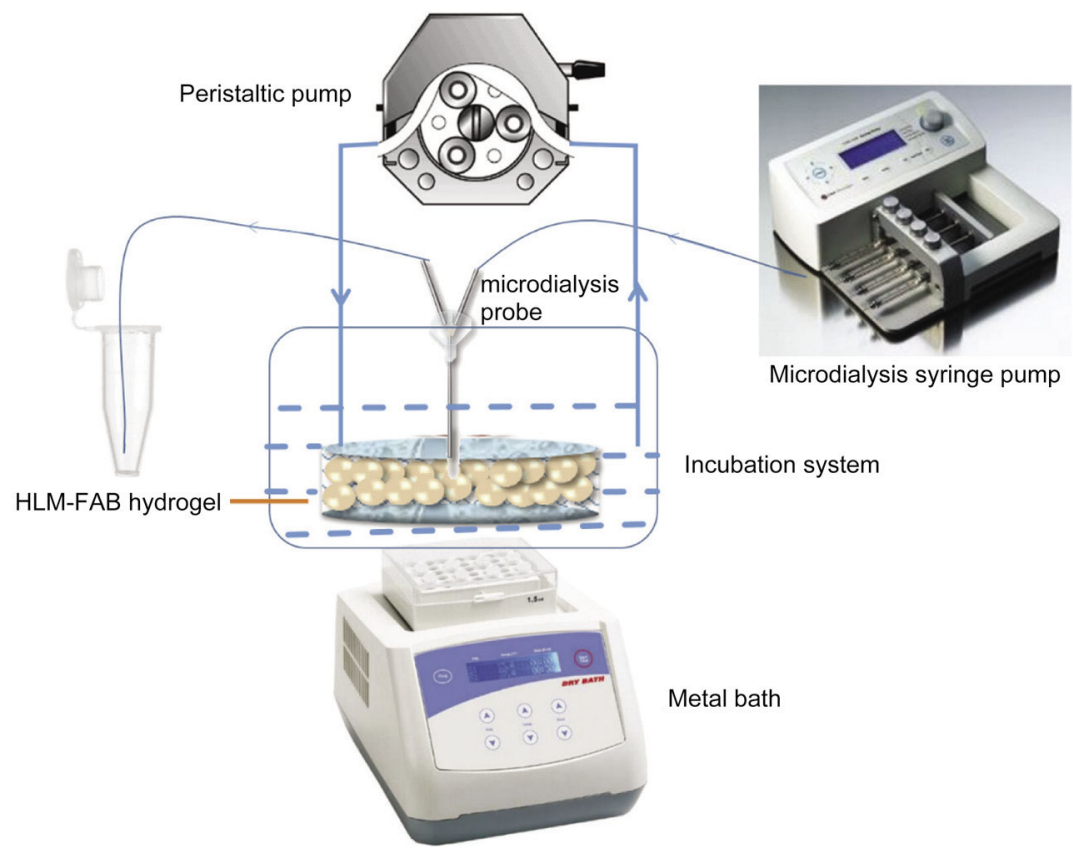

Figure 1. Schematic of the Bio-PK system, consisting of a peristaltic pump, incubation systems and microdialysis systems. HLMs encapsulated in the FAB hydrogel were placed in the incubation system, which was placed in a thermostatic metal bath. The microdialysis system consisted of a microdialysis syringe pump and microdialysis probes.

\section{Microdialysis sampling}

Microdialysis sampling techniques were integrated into the Bio-PK system to better obtain samples from the FAB hydrogels in real time. The microdialysis systems consisted of a microdialysis syringe pump (CMA/400, CMA, Stockholm, Sweden) and microdialysis probes. The dialysis probes $(2 \mathrm{~mm}$ in length) have a molecular weight cut-off of $6 \mathrm{kDa}$ (Microbiotech/se AB, Stockholm, Sweden). The substance used as the membrane material was PES (polyether sulphone). The microdialysis probe was inserted into the FAB hydrogel. Thereafter, the probes were perfused with Ringer's solution at a flow rate of $1 \mu \mathrm{L} / \mathrm{min}$, using the CMA microdialysis syringe pump for delivery of the perfusion solution. The perfusate was passed through a $0.22-\mu \mathrm{m}$ nylon filter before use. The perfusion fluid entered the probe through the inlet tubing at a flow rate of $1 \mu \mathrm{L} / \mathrm{min}$, passed the membrane and was then transported through the outlet tubing, collected in a microtube and analyzed by LC-MS/MS.

\section{Recovery assessment of microdialysis probes}

The recovery for the microdialysis probe was determined using the no net flux method $(\mathrm{NNF})^{[23-25]}$. The microdialysis probe was inserted into the $\mathrm{FAB}$ hydrogel from the incubation system of the Bio-PK system at $37^{\circ} \mathrm{C}$ containing tolbutamide (200 $\mu \mathrm{mol} / \mathrm{L}, 100 \mu \mathrm{mol} / \mathrm{L}$ and $50 \mu \mathrm{mol} / \mathrm{L})$ and was perfused at $1 \mu \mathrm{L} / \mathrm{min}$ with Ringer's solution containing different concentrations of tolbutamide (5, 20, 80, 150, 250 and $300 \mu \mathrm{mol} / \mathrm{L}$, $\mathrm{Cp})$. The dialysate samples $(\mathrm{Cd})$ were collected continuously every 15 minutes $(n=3)$ for each concentration of perfusion solution and were analyzed by LC-MS/MS. Linear regression analysis was performed with the perfusion solution $(\mathrm{Cp})$ as the abscissa and the concentration difference between the microdialysate samples and the perfusion solution $(\mathrm{Cd}-\mathrm{Cp})$ as the ordinate. The slope was the relative recovery (RR) of the probe, and the intercept of the straight line and the $\mathrm{X}$ axis was the free drug concentration (Cf).

The recovery of 4-hydroxytolbutamide was obtained in a similar manner as the determination of tolbutamide recovery. The concentration of 4-hydroxytolbutamide in the vial of the dialysis medium was $10 \mu \mathrm{mol} / \mathrm{L}$, and six perfusates contained different concentrations of 4-hydroxytolbutamide (1, 10, 20, 40, 80 and $100 \mu \mathrm{mol} / \mathrm{L})$.

\section{Diffusion coefficients}

To determine the diffusion coefficients, the FAB hydrogel containing microsomes with a final concentration of $0.5 \mathrm{mg} / \mathrm{mL}$ was placed in the incubation system. The microdialysis probe was inserted into the FAB hydrogel. Thereafter, the probes were perfused with Ringer's solution at a flow rate of 1 $\mu \mathrm{L} / \mathrm{min}$. Following the addition of $200 \mu \mathrm{mol} / \mathrm{L}$ tolbutamide, the dialysate samples were collected at 5, 10, 25, 40, 55, 70, 85, 100 and $120 \mathrm{~min}$ and were analyzed by LC-MS/MS.

\section{Flow rate for the Bio-PK metabolic system}

To determine the final flow rate of the Bio-PK metabolic system, different flow rates were set to examine the release of microsomes from the FAB hydrogel and the production of metabolites.

HLMs encapsulated in the FAB hydrogel (at a final concentration of $0.5 \mathrm{mg} / \mathrm{mL}$ ) were placed in the incubation system at $37^{\circ} \mathrm{C}$, and the total circulating fluid volume was $3 \mathrm{~mL}$. The system was circulated for $24 \mathrm{~h}$ with a flow rate of $0 \mathrm{~mL} / \mathrm{min}$, 
$21 \mathrm{~mL} / \mathrm{min}, 42 \mathrm{~mL} / \mathrm{min}, 63 \mathrm{~mL} / \mathrm{min}$ or $85 \mathrm{~mL} / \mathrm{min}$, and $50 \mu \mathrm{L}$ of circulating fluid was collected at $0.5,1,2,4,6,8$ and $24 \mathrm{~h}$ for protein quantitation using the BCA Protein Assay Kit.

To further confirm the flow rate, $200 \mu \mathrm{mol} / \mathrm{L}$ tolbutamide and $1 \mathrm{mmol} / \mathrm{L}$ NADPH were added to the same incubation system above to detect metabolites with a flow rate of 5 $\mathrm{mL} / \mathrm{min}, 10 \mathrm{~mL} / \mathrm{min}, 20 \mathrm{~mL} / \mathrm{min}$ or $40 \mathrm{~mL} / \mathrm{min}$.

\section{Metabolism of tolbutamide in the Bio-PK metabolic system}

Following the above description to construct the Bio-PK metabolic system, the volume and flow rate of the total circulating fluid were $3 \mathrm{~mL}$ and $10 \mathrm{~mL} / \mathrm{min}$, respectively. FAB hydrogel was immersed in HLMs for $48 \mathrm{~h}$ at $4{ }^{\circ} \mathrm{C}$ and was immediately mixed with PBS buffer containing tolbutamide and NADPH (1 $\mathrm{mmol} / \mathrm{L}$ ) to a final protein concentration of $0.5 \mathrm{mg} / \mathrm{mL}$. The incubation system was preincubated to $37^{\circ} \mathrm{C}$ for $5 \mathrm{~min}$ in a thermostatic metal bath. The cyclic reactions were initiated with the addition of tolbutamide stock solutions (50, 100 and $200 \mu \mathrm{mol} / \mathrm{L}$ final concentrations). Samples were gently mixed and maintained at $37^{\circ} \mathrm{C}$ for $4 \mathrm{~h}$. Samples of FAB hydrogel and circulating fluid were taken separately after incubations of 0 , $20,40,60,80,100,120,180$, and $240 \mathrm{~min}$. The samples of circulating fluid were immediately added with 2 times methanol containing the internal standard (IS; phenacetin) and mixed for $2 \mathrm{~min}$ on a vortex mixer to terminate the reaction. The solution was centrifuged at $138000 \times g$ for $10 \mathrm{~min}$. The supernatant was transferred to a separate container for LC-MS/MS analysis. The dialysate samples from the FAB hydrogel were directly transferred to microtubes and analyzed by LC-MS/ MS within $48 \mathrm{~h}$.

\section{Mathematical model}

Based on the circulating perfusion and mass balance of the Bio-PK system, a mathematical model was developed to describe the above dynamic process and was fitted to the clearance $\left(\mathrm{CL}_{\mathrm{g}}\right)$ in FAB hydrogel using MATLAB (MathWorks, Inc, Natick, MA, USA). The model considers factors such as diffusion in the FAB hydrogels, the interchange between the FAB hydrogels and the circulating fluid, HLM protein binding and dynamic circulation. The in vitro intrinsic clearance $\left(\mathrm{CL}_{\mathrm{int}}\right)$ in human is based on the assumption that HLMs per mass unit have the same metabolic capacity. A scheme of the mathematical model is shown in Figure 2. The relevant formula is as follows:

$$
\begin{gathered}
\frac{d C_{m}(t) V_{m}}{d t}=D\left(C_{g}(\mathrm{t})-f_{m} C_{m}(t)\right)+(1-\gamma) Q C_{m}\left(t-\tau_{1}\right)-\gamma Q C_{u}(t)-Q C_{m}(t) \\
\frac{d C_{g}(t) V_{g}}{d t}=-D\left(C_{g}(\mathrm{t})-f_{m} C_{m}(t)\right)+\gamma Q C_{m}\left(t-\tau_{1}\right)-C L_{g} C_{g}(t)-\gamma Q C_{u}\left(t-\tau_{2}\right) \\
C_{\mathrm{u}}(\mathrm{t})=C_{\mathrm{g}}\left(t+\tau_{2}\right)-C L_{\mathrm{g}} C_{\mathrm{g}}\left(t+\tau_{2}\right) \tau_{2} / V_{\mathrm{g}} \\
\frac{A_{\mathrm{MTB}}(t)}{d t}=C L_{\mathrm{g}} C_{\mathrm{g}}(t)
\end{gathered}
$$

where $C_{\mathrm{m}}$ and $C_{\mathrm{g}}$ are the concentration and $V_{\mathrm{m}}$ and $V_{\mathrm{g}}$ the volume of substrate in the circular medium and FAB hydrogel,

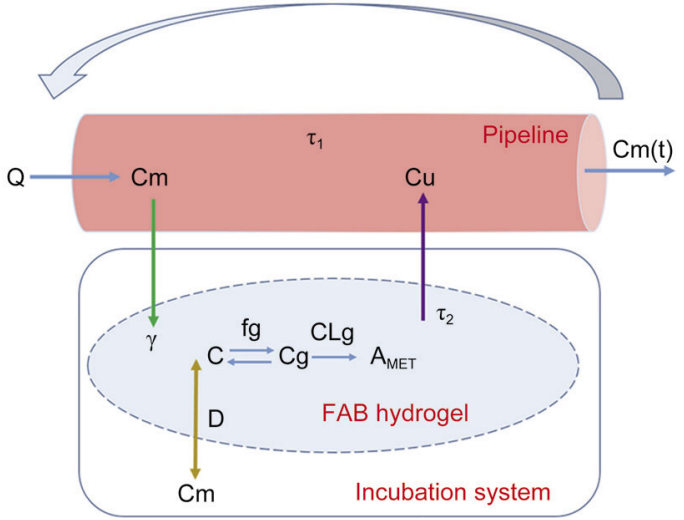

Figure 2. Dynamic disposition scheme of the Bio-PK system. $C_{m}$, concentration of substrate in circular medium; $C_{g}$, concentration of substrate in FAB hydrogel; $C_{u}$, concentration of substrate flow out of FAB hydrogel; $A_{\text {MTB }}$, amount of metabolites; $Q$, flow rate; $D$, diffusion coefficients; $f_{\mathrm{g}}$, free fraction; $\mathrm{\gamma}$, proportion flowing into FAB hydrogel; $\tau_{1}$, time flowing through pipeline; $\tau_{2}$, time flowing through $\mathrm{FAB}$ hydrogel; $\mathrm{CL}_{\mathrm{g}}$, clearance in FAB hydrogel.

respectively; $C_{u}$ is the substrate concentration that came from the hydrogel due to circulation; and the amount of metabolites is expressed by AMTB. $Q$ is the flow rate of the peristaltic pump; $D$ is the diffusion coefficient; $f_{\mathrm{m}}$ is the free fraction; $\gamma$ is the crossover proportion from one end of the pipe into the FAB hydrogel; $\tau_{1}$ is the time from one end to the other in the pipeline of the peristaltic pump; and $\tau_{2}$ is the migration time in the gel.

\section{PBPK model development}

To better verify the Bio-PK system, a PBPK model was developed to predict the PK profile and CL of tolbutamide using the Simcyp ${ }^{\mathrm{TM}}$ population-based ADME simulator (V15; Simcyp Limited, Sheffield, UK). The PBPK model was simulated by integrating the in silico and in vitro data of the drug in healthy volunteer population databases from the Simcyp simulator. The Monte Carlo method allows the random generation of a unique set of physiological parameters for each virtual subject, such as anatomic, demographic, and tissue-specific parameters. The variation in each variable was generated by random sampling of each variable within its defined distribution for each simulated virtual subject. The default trial designed by Simcyp was selected to build the model for tolbutamide. A virtual population of 100 healthy volunteers (10 trials with 10 subjects each), aged 20-50 years with a female/male ratio of 0.5 , was used to simulate PK following single oral doses of 500 mg of tolbutamide.

A minimal PBPK distribution model with tissue partition coefficients was predicted by the Rodgers method ${ }^{[26]}$, and a first-order absorption model was used to describe the distribution and absorption. The elimination process was characterized by the intrinsic clearance of CYP2C 9 and by kidney clearance.

The accuracy of the PBPK model was assessed by compar- 
Table 1. In vitro microdialysate recoveries for tolbutamide and 4-hydroxytolbutamide.

\begin{tabular}{ll}
\hline Concentration $(\mu \mathrm{mol} / \mathrm{L})$ & Recovery $(\%)$ \\
\hline Tolbutamide & \\
200 & $21.88 \pm 1.16$ \\
100 & $26.50 \pm 1.02$ \\
50 & $22.83 \pm 0.68$ \\
Average & $23.74 \pm 1.35$ \\
$4-$ Hydroxytolbutamide & \\
8 & $33.46 \pm 0.16$ \\
\hline
\end{tabular}

Data are expressed as mean \pm standard deviation $(n=3)$.

ing the ratio between the predicted and observed pharmacokinetic parameters (fold error), together with visual inspection. If observed $>$ predicted, the fold error $=$ observed/predicted; otherwise, fold error=predicted/observed. A model with a fold error of less than two is generally considered a precise model ${ }^{[27-31]}$.

\section{Results}

\section{In vitro relative recovery of probes}

The in vitro recoveries of probes for tolbutamide and 4-hydroxytolbutamide are shown in Table 1. The actual concentration of tolbutamide and 4-hydroxytolbutamide in the FAB hydrogel could be corrected by an average recovery of $23.74 \%$ and $33.46 \%$, respectively.

\section{Diffusion coefficients}

The diffusion coefficients of tolbutamide from the medium into FAB hydrogel are shown in Figure 3. A final concentration of $0.5 \mathrm{mg} / \mathrm{mL}$ microsomes was encapsulated in $300 \mu \mathrm{L}$ of FAB hydrogel. Following the addition of drug, tolbutamide is rapidly diffused into the $\mathrm{FAB}$ hydrogel due to the leakage effect between the hydrogel and the medium; equilibrium was reached after $70 \mathrm{~min}$ as the reaction progressed. Therefore, the diffusion coefficient of tolbutamide was estimated as 0.004 $\mathrm{mL} / \mathrm{min}$.

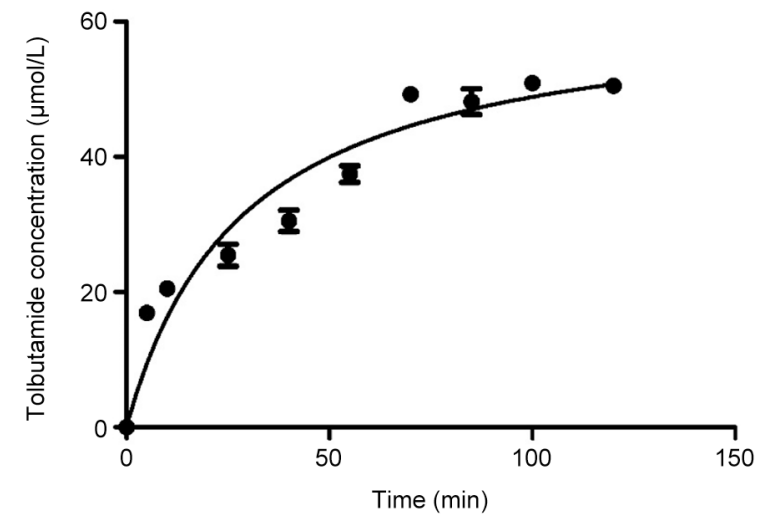

Figure 3. Diffusion of tolbutamide from medium to FAB hydrogel; $n=3$, mean \pm SD.

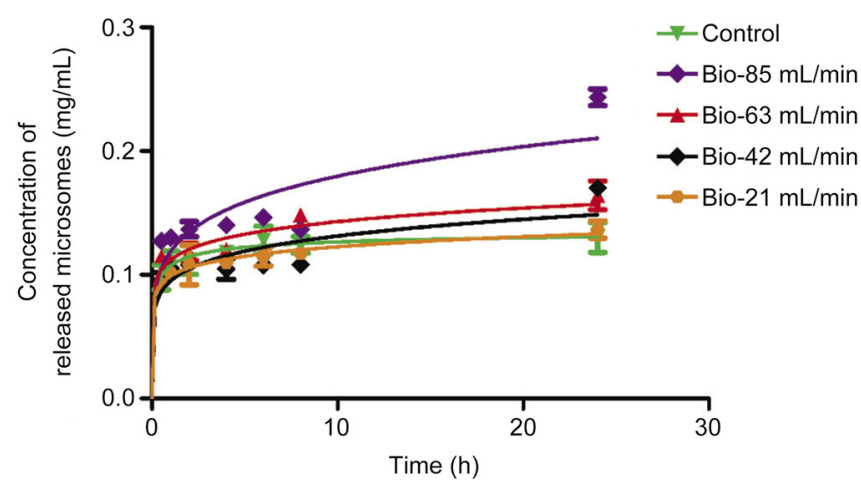

Figure 4. Release of HLM protein from FAB hydrogel in the Bio-PK metabolic system under various flow rates, compared to the control group under static conditions; $n=3$, mean \pm SD.

\section{Flow rate for the Bio-PK metabolic system}

The release of microsomal protein from the FAB hydrogel was determined under different flow rates, as shown in Figure 4. Data in the control group indicate that the release of microsomal protein from the FAB hydrogel is in a static state.

With the increase in flow rate, the release of microsomal protein from the FAB hydrogel showed a tendency to increase, indicating that it was gradually washed out of the hydrogel. When the flow rate was $21 \mathrm{~mL} / \mathrm{min}$, the release of microsomes was consistent with that of the control group. The concentration of microsomal protein increased slightly in the first few minutes and then immediately reached the plateau stage, indicating that the microsomal proteins are firmly locked inside the FAB hydrogel and remain stable for a long period. However, when the flow rate was above $21 \mathrm{~mL} / \mathrm{min}$, the release of microsomes was higher than in the control group. With increasing flow rate, the time at which the microsomes washed out of the hydrogel gradually advanced. The microsomes washed out of the hydrogel after approximately $12 \mathrm{~h}$ at 42 $\mathrm{mL} / \mathrm{min}$. At a flow rate of $63 \mathrm{~mL} / \mathrm{min}$, the time advanced to 6 $\mathrm{h}$, and when the flow rate increased to $85 \mathrm{~mL} / \mathrm{min}$, the microsomes were rinsed out first.

Based on the above results, we tested four flow rates to further examine the metabolic status of tolbutamide as the flow

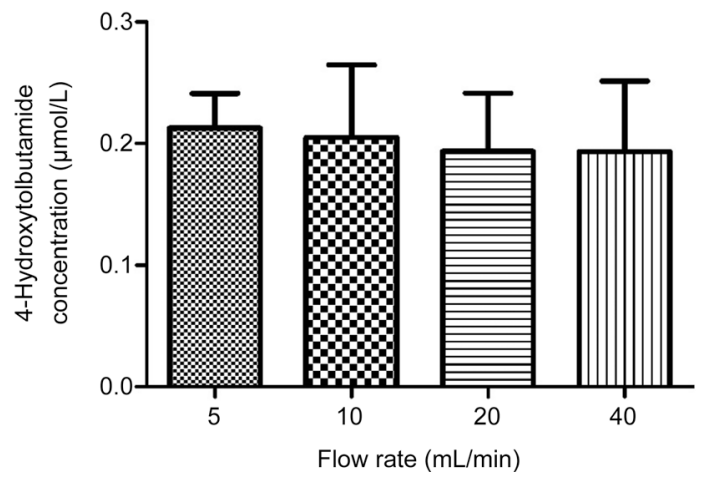

Figure 5. Metabolic status of tolbutamide at various flow rates. $n=3$, mean \pm SD. 
rate varied. The data are shown in Figure 5. In the figure, the metabolites of tolbutamide formed are basically the same at different flow rates, consistent with our expectation. This indicates that the microsomes are well encapsulated by the FAB hydrogel at a flow rate of less than $42 \mathrm{~mL} / \mathrm{min}$. Considering the destruction of the hydrogel and the long-term decrease in loading capacity at high flow rates, a flow rate of $10 \mathrm{~mL} / \mathrm{min}$ was selected for the Bio-PK system.

\section{Tolbutamide metabolism in the Bio-PK metabolic system}

After successful construction of the Bio-PK metabolic system, various concentrations of tolbutamide were placed in the system for circulating incubation. The related results are shown in Figures 6, 7 and 8. In the Bio-PK system, the production of 4-hydroxytolbutamide consistently increased over time and was certainly correlated with the concentration of the substrate. As the reaction progressed, the amount of tolbutamide in the FAB hydrogel gradually increased and finally reached the saturation level. The volume of the FAB hydrogel was small, resulting in less impact on the substrate concentration in the circulating fluid after the substrate entered the FAB hydrogel over time. Therefore, the concentration of tolbutamide in the circulating fluid was essentially unchanged. The

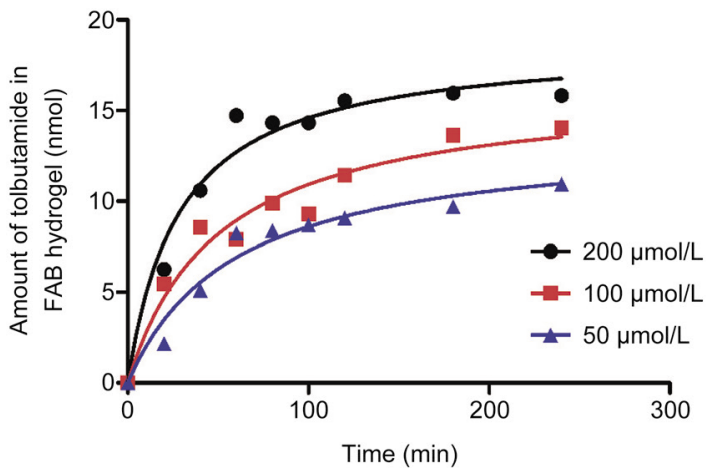

Figure 6. Different concentrations of tolbutamide over time in the $F A B$ hydrogel obtained using the Bio-PK metabolic system; $n=3$, mean \pm SD.

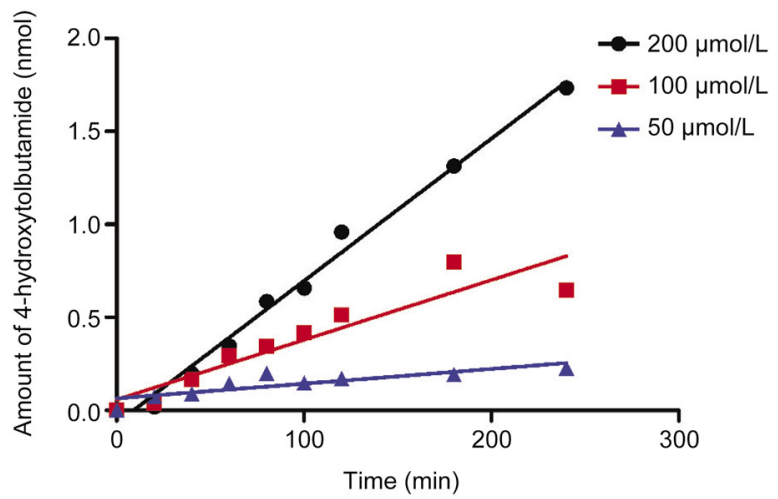

Figure 7. Amount of 4-hydroxytolbutamide formed by different concentrations of tolbutamide over time obtained using the Bio-PK metabolic system; $n=3$, mean \pm SD.

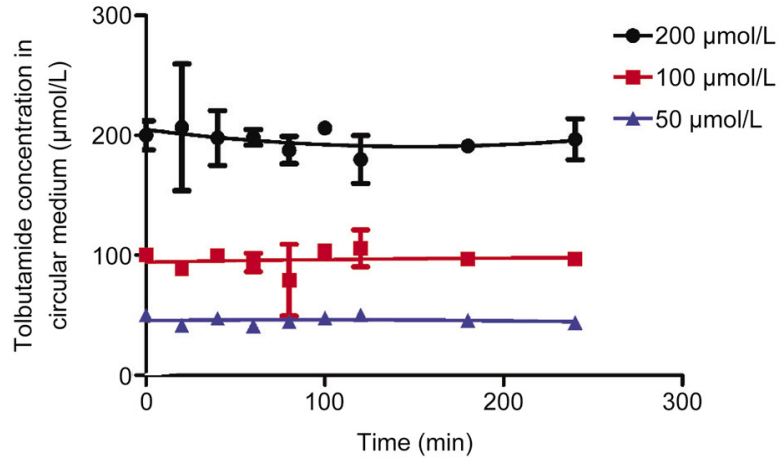

Figure 8. Concentration of tolbutamide over time in medium obtained using the Bio-PK metabolic system; $n=3$, mean \pm SD.

above data will be applied to the mathematical model to fit tolbutamide clearance.

\section{Mathematical model}

To better describe the convection-diffusion-reaction characteristics inside the Bio-PK system, a mathematical model was developed using MATLAB by optimizing the discrete model of the parameter values, considering the experimental data and assuming a relative error model. In this model, $\mathrm{CL}_{\mathrm{g}}$ is the intrinsic substrate clearance in the FAB hydrogel. Based on the metabolism of tolbutamide in the Bio-PK metabolic system, $\mathrm{CL}_{\mathrm{g}}$ was fitted to $0.0102 \mathrm{~mL} / \mathrm{min}$ for $200 \mu \mathrm{mol} / \mathrm{L}, 0.009$ $\mathrm{mL} / \mathrm{min}$ for $100 \mu \mathrm{mol} / \mathrm{L}$, and $0.0072 \mathrm{~mL} / \mathrm{min}$ for $50 \mu \mathrm{mol} / \mathrm{L}$. Thus, the corresponding intrinsic clearance of tolbutamide was $6.8(200 \mu \mathrm{mol} / \mathrm{L}), 6.0(100 \mu \mathrm{mol} / \mathrm{L})$ and $4.8(50 \mu \mathrm{mol} / \mathrm{L})$ $\mu \mathrm{L} / \mathrm{min} / \mathrm{mg}$, respectively.

\section{PBPK model for tolbutamide}

The PBPK model of tolbutamide was built based on the parameters in Table 2. The simulated PK profiles after oral doses of $500 \mathrm{mg}$ of tolbutamide are shown in Figure 9. Compared to the intrinsic clearance obtained from the previous traditional incubation method, a good match for the PK profile can be predicted by integrating the intrinsic clearance of tolbutamide obtained from the Bio-PK system into the PBPK model. The simulated $C_{\max }$ AUC and $T_{\max }$ of tolbutamide, based on the average intrinsic clearance $(5.87 \mu \mathrm{L} / \mathrm{min} / \mathrm{mg})$, were 36331 $\mathrm{ng} / \mathrm{mL}, 519080 \mathrm{ng} \cdot \mathrm{h} / \mathrm{mL}$, and $3.6 \mathrm{~h}$, respectively. All were within 2-fold error of the observed results $\left(C_{\max }=33500 \mathrm{ng} / \mathrm{mL}\right.$, AUC $=483223 \mathrm{ng} \cdot \mathrm{h} / \mathrm{mL}$ and $\left.T_{\max }=4.1 \mathrm{~h}\right)^{[32]}$ (Table 3). The literature and predicted in vivo $\mathrm{CL}$ values for tolbutamide are shown in Table 4 . The predicted in vivo CL values for tolbutamide were $1.26 \mathrm{~L} / \mathrm{h}$ for a $200 \mu \mathrm{mol} / \mathrm{L}$ incubation, $1.11 \mathrm{~L} / \mathrm{h}$ for a $100 \mu \mathrm{mol} / \mathrm{L}$ incubation and $0.89 \mathrm{~L} / \mathrm{h}$ for a $50 \mu \mathrm{mol} / \mathrm{L}$ incubation. The mean in vivo $\mathrm{CL}$ of tolbutamide predicted by the PBPK model using the clearance obtained from the Bio-PK system was $1.09 \mathrm{~L} / \mathrm{h}$, in good agreement with (<2-fold error) the observed results (mean $\mathrm{CL}=0.84 \mathrm{~L} / \mathrm{h})^{[33-38]}$. However, the predicted mean $\mathrm{CL}$ obtained from traditional incubation was $0.38 \mathrm{~L} / \mathrm{h}^{[39,40]}$, indicating that the Bio-PK system can better predict metabolic behavior in vivo and obtain more accurate in 
Table 2. Parameters for tolbutamide used in PBPK modeling.

\begin{tabular}{|c|c|c|}
\hline \multirow[t]{2}{*}{ Parameter } & \multicolumn{2}{|c|}{ Tolbutamide } \\
\hline & Value & References/Comments \\
\hline Mol weight $(\mathrm{g} / \mathrm{mol})$ & 270.3 & Drug bank \\
\hline $\log P_{o: w}$ & 2.34 & Drug bank \\
\hline pKa & 5.16 & Drug bank \\
\hline$B / P$ & 0.55 & {$[55]$} \\
\hline$f_{\mathrm{u}, \mathrm{p}}$ & 0.054 & {$[56]$} \\
\hline$f_{\mathrm{a}}$ & 0.93 & [34] \\
\hline$k_{\mathrm{a}}\left(\mathrm{h}^{-1}\right)$ & 0.52 & {$[34]$} \\
\hline $\mathrm{Q}_{\text {Gut }}(\mathrm{L} / \mathrm{h})$ & 5.73 & Predicted \\
\hline Permeability & 0.95 & Predicted \\
\hline PSA $\left(\AA^{2}\right)$ & 83.65 & Pubchem \\
\hline HBD & 2 & Pubchem \\
\hline$V_{\mathrm{ss}}(\mathrm{L} / \mathrm{kg})$ & 0.105 & Predicted with Rogers method \\
\hline $\mathrm{CL}_{\mathrm{R}}$ & 0.0019 & {$[54]$} \\
\hline Enzyme & CYP2C9 & Metabolite: 4-hydroxytolbutamide \\
\hline $\mathrm{CL}_{\text {int }}(\mu \mathrm{L} / \mathrm{min}$ & 1.87 & {$[39]$} \\
\hline per milligram) & 2.28 & [40] \\
\hline & $\begin{array}{l}6.80,6.00,4.80 \\
(m e a n=5.87)\end{array}$ & Bio-PK system \\
\hline$f_{u, \text { mic }}$ & 0.97 & {$[57]$} \\
\hline
\end{tabular}

$\mathrm{B} / \mathrm{P}$, blood-to-plasma ratio; $f_{\mathrm{u}, \mathrm{p}}$, free fraction in plasma; $f_{\mathrm{a}}$, fraction of dose absorbed; $k_{a}$, first-order absorption rate constant; $V_{s s}$, steady-state volume of distribution; $f_{u, m i c}$, free fraction in liver microsome; $\mathrm{CL}_{\text {int }}$, intrinsic clearance; $\mathrm{CL}_{R}$, renal clearance; $Q_{\text {Gut }}$, flow rate for overall delivery of drug to the gut; PSA, polar surface area; HBD, hydrogen bond donors.

vitro data.

\section{Discussion}

Liver metabolism is commonly considered the major determinant in drug discovery and development. Many in vitro drug metabolic studies have already been developed and applied to understand biotransformation. These provide some guidance for the screening and development of drugs, as well as the elucidation of metabolic mechanisms and pathways ${ }^{[9]}$. However, these methods have disadvantages, resulting in inconsistencies between in vivo and in vitro experiments. A major factor is that they are static systems that do not consider the transport process in the liver.

Based on our previous studies, a dynamic Bio-PK metabolic

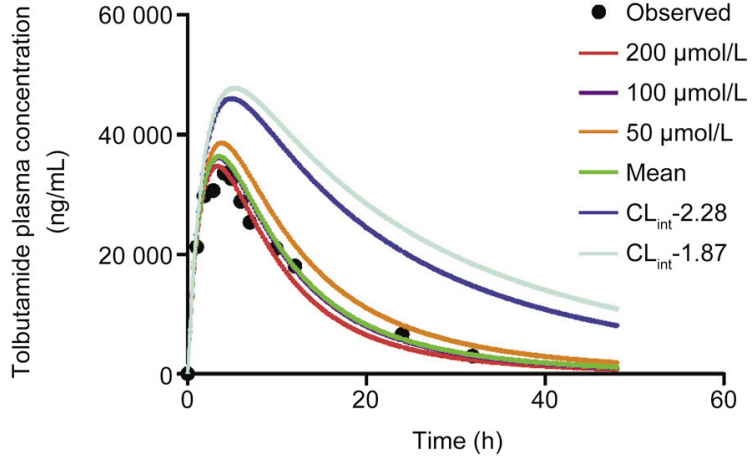

Figure 9. Predicted and observed mean plasma concentration-time profiles of tolbutamide after a single oral dose of $500 \mathrm{mg}$ of tolbutamide. The solid lines represent the mean plasma concentrations predicted by integrating various in vitro intrinsic clearances; red, purple, orange and green lines represent the plasma concentration simulated by integration of the intrinsic clearance fitted using the Bio-PK system, whereas blue and pale blue lines represent the integration of the intrinsic clearance obtained from the traditional incubation method ${ }^{[39,40]}$. Symbols represent mean observed data ${ }^{[32]}$.

system that consists of a peristaltic pump and an incubation system containing a rat microsome-encapsulated FAB hydrogel is highly expected to be a new tool for in vitro metabolic study $^{[21,22]}$. However, the ultimate goal of drug research is focused on human studies. It is often desirable to estimate clearance in humans from measurements performed in vitro or in other species. A specific probe substrate is more suitable and can more clearly illustrate the dynamic system. Tolbutamide- a classical probe substrate for CYP2C9 based on a representative list of preferred and acceptable in vitro probe substrates recommended by FDA guidance-was chosen as the model drug. Many in vitro and in vivo studies have enabled comparison and validation of the dynamic system. In addition, it has been reported that the drug has a low extraction rate $^{[41,42]}$, and most current incubation times are short (approximately $30 \mathrm{~min}$ ), which affects tolbutamide metabolism. The HLMs encapsulated in the FAB hydrogel can better extend microsomal activity at long incubation times. In the dynamic system, tolbutamide quickly diffused in the hydrogel, the accumulation of metabolite increased linearly with time, the substrate and metabolites were accurately detected, and the

Table 3. Observed versus predicted PK data (AUC, $C_{\max }$ and $T_{\max }$ ) of tolbutamide in the PBPK model of tolbutamide study.

\begin{tabular}{|c|c|c|c|c|c|c|c|}
\hline \multirow{2}{*}{$\begin{array}{l}\mathrm{CL}_{\text {int }}(\mu \mathrm{L} / \mathrm{min} \\
\text { per milligram })\end{array}$} & \multicolumn{2}{|c|}{$\mathrm{AUC}(\mathrm{ng} / \mathrm{mL} \cdot \mathrm{h})$} & \multicolumn{2}{|c|}{$C_{\max }(\mathrm{ng} / \mathrm{mL})$} & \multicolumn{2}{|c|}{$T_{\max }(\mathrm{h})$} & \multirow[t]{2}{*}{ References/Comments } \\
\hline & Predicted & Observed $^{[32]}$ & Predicted & Observed $^{[32]}$ & Predicted & Observed $^{[32]}$ & \\
\hline 6.80 & 453399 & & 34671 & & 3.37 & & Bio-system for $200 \mu \mathrm{mol} / \mathrm{L}$ \\
\hline 6.00 & 508844 & & 36079 & & 3.37 & & Bio-system for $100 \mu \mathrm{mol} / \mathrm{L}$ \\
\hline 4.80 & 620661 & 483223 & 38564 & 33500 & 3.87 & 4.1 & Bio-system for $50 \mu \mathrm{mol} / \mathrm{L}$ \\
\hline 5.87 & 519080 & & 36331 & & 3.62 & & Bio-system for Mean \\
\hline 2.28 & 1105571 & & 45984 & & 4.83 & & {$[40]$} \\
\hline 1.87 & 1256410 & & 47719 & & 5.33 & & [39] \\
\hline
\end{tabular}


Table 4. The observed and simulated CL for tolbutamide.

\begin{tabular}{llll}
\hline Parameter & \multicolumn{2}{c}{ Tolbutamide } & References/Comments \\
& Value & Mean & \\
\hline $\mathrm{CL}(\mathrm{L} / \mathrm{h})$ & 1.05 & & {$[33]$} \\
& 0.79 & 0.84 & {$[33]$} \\
& 0.80 & & {$[33]$} \\
& 0.75 & & {$[37]$} \\
0.73 & & {$[38]$} \\
& 0.79 & & {$[36]$} \\
0.85 & & {$[34]$} \\
& 0.93 & & {$[35]$} \\
0.34 & 0.38 & Traditional incubation \\
& 0.42 & & Traditional incubation \\
& 1.26 & & Bio-system for $200 \mu \mathrm{mol} / \mathrm{L}$ \\
& 1.11 & \multirow{2}{*}{1.09} & Bio-system for $100 \mu \mathrm{mol} / \mathrm{L}$ \\
0.89 & & Bio-system for $50 \mu \mathrm{mol} / \mathrm{L}$ \\
\hline
\end{tabular}

data could be fitted with a high degree of correlation. Finally, we also sought to further verify whether the system can be applied to and promoted in different species to provide the basis for further optimization and application of the system.

In this Bio-PK metabolic system, HLMs were encapsulated in the FAB hydrogel as a metabolic element. To better monitor the drug concentration in the FAB hydrogels in realtime, microdialysis ${ }^{[43]}$, a semi-invasive sampling technique whose principle is dialysis, was integrated into the Bio-PK system. For analysis of the small sample volumes with low concentrations of analytes frequently present in microdialysis samples ${ }^{[44]}$, LC-MS/MS was used to measure analyte concentrations in the microdialysates ${ }^{[45]}$. The free drug is more likely to reach the target organ from the vascular compartment and is considered the pharmacologically active portion. Furthermore, plasma and microsomal protein binding plays an important role in pharmacokinetic processes, especially in the distribution and elimination of $\mathrm{drug}^{[46]}$. The main advantage of microdialysis is to exclude proteins from the microdialysate, which stops enzymatic degradation, terminates microsome reactions, and makes sample preparation redundant. Therefore, all microdialysis characteristics are very suitable for use in the Bio-PK metabolic system and will further refine the system.

In fact, the dialysis process is not in complete equilibrium, and the concentration of the drug in the dialysate obtained by microdialysis is different from that in the periprobe fluid. It is mainly the concentration of the drug around the semipermeable membrane of the probe. Thus, the in vitro recovery of the probe was used to calibrate the concentration difference. Microdialysis probe recovery was calibrated using the no-net-flux method. No difference in recovery values was observed for the different concentrations of tolbutamide, indicating that the recoveries from the microdialysis probes in the FAB hydrogel are independent of tolbutamide concentration. The recovery of 4-hydroxytolbutamide in the FAB hydrogel was higher than that of tolbutamide, which may be attributed to the metabolism of xenobiotics. This metabolism usually results in compounds that are more polar than the parent drug (reduction in $\log \mathrm{D})$, which tends to increase the affinity for drug perfusate ${ }^{[47]}$. The perfusate of the microdialysis probe is typically aqueous, such as phosphate buffer (PBS), Ringer's solution and anticoagulant citrate dextrose (ACD solution) $)^{[48]}$. Therefore, microdialysis technology is more suitable for the sampling of polar components, and it is difficult to the high hydrophobic and protein binding components ${ }^{[49,50]}$. Thus, it is reasonable to expect that 4-hydroxytolbutamide can more easily pass through the membrane compared to tolbutamide.

The average fasted-state hepatic blood flow rate in humans is $90 \mathrm{~L} / \mathrm{h}(1.5 \mathrm{~L} / \mathrm{min})$. This increases in the fed state to $120 \mathrm{~L} / \mathrm{h}(2 \mathrm{~L} / \mathrm{min})^{[51]}$. When such a high flow rate is applied to in vitro studies, it leads to a higher requirement for the pump; importantly, the FAB hydrogel can be easily destroyed, which may easily lead to the loss of enzyme activity. Thus, a suitable flow rate for the system must be determined. This flow rate should ensure that the FAB hydrogel is not destroyed and that microsomal proteins are firmly locked inside the FAB hydrogel and remain stable for a long period. The release of HLM protein from the FAB hydrogel under different flow rates shows that the HLMs are substantially encapsulated in the $\mathrm{FAB}$ hydrogel when the flow rate is less than or equal to $21 \mathrm{~mL} / \mathrm{min}$. The formation of metabolites further supports the encapsulation capacity and integrity of the FAB hydrogels. Although the FAB hydrogel remains stable and has good loading capacity at $42 \mathrm{~mL} / \mathrm{min}$ in the short term, the HLMs will still be washed out over time. Thus, an average flow rate of $10 \mathrm{~mL} / \mathrm{min}$ was used in the Bio-PK metabolic system to study human metabolism.

Quantitative analysis of the liver metabolism reaction was demonstrated by combining a mathematical modeling approach and experimental analysis. These complex dynamic processes for the Bio-PK metabolic system, including diffusion, microsomal protein binding, transport and reaction and the substrate interchange between the FAB hydrogel and the circular medium, were described. However, traditional in vitro systems cannot reproduce this aspect of liver metabolism. A diffusion coefficient, D, is used to calculate the amount of drug in the hydrogel at each time step. Partitioning is assumed to be instantaneous, and the diffusion coefficient can be estimated from the experiment. If diffusion is high, the amount of drug that partitions into the hydrogel will be limited by the blood flow rate (perfusion rate) through the hydrogel. In the dynamic system, tolbutamide quickly diffused in the hydrogel, and its metabolic clearance was far below the blood flow rate. As mentioned above, the flow rate of the BioPK metabolic system ensures that the FAB hydrogel is intact and well encapsulated for HLMs; thus, $f_{\mathrm{m}}$ is assumed to be 1 in the model. An average $\mathrm{CL}_{\mathrm{g}}$ of $0.0088 \mathrm{~mL} / \mathrm{min}$ was fitted. The intrinsic clearance was integrated into a PBPK model to predict the PK profile and in vivo clearance in humans.

A mini-PBPK distribution model and a first-order absorption model were used for a good description of the tolbutamide PK profile. A distribution of $0.105 \mathrm{~L} / \mathrm{h}$ was estimated using the 
mechanistic tissue composition equation ${ }^{[26]}$ set in Simcyp. This result was in good agreement with the observed results (mean $\left.V_{\mathrm{ss}}=0.103 \mathrm{~L} / \mathrm{h}\right)^{[34-36]}$. In human beings, tolbutamide is metabolized almost exclusively by CYP2C9 to hydroxytolbutamide. Clearance of tolbutamide from the body is equal to metabolic clearance plus the renal clearance of unchanged drug ${ }^{[32,35,52]}$. Thus, in the PBPK model, elimination was included in the integration of in vitro intrinsic clearance and renal clearance. Based on the in vitro data and the mechanisms mentioned above, an $f_{\mathrm{e}}$ (fraction of total body clearance via renal excretion) of $0.26 \%$ and an $f_{\mathrm{m}}$ (fraction of substrate clearance due to each specific enzyme) of 99.74 were reasonably predicted by the PBPK model, which integrated the average intrinsic clearance from the Bio-PK metabolic system. Studies have shown that the $f_{\mathrm{m}}$ for tolbutamide is $1^{[53]}$. The urinary excretion of tolbutamide at different times is also reasonably predicted. The predicted cumulative urinary recovery of tolbutamide was $1.3 \mu \mathrm{mol}$ between 0 and $6 \mathrm{~h}$ (observed $1.5 \mu \mathrm{mol}$ ), $2.4 \mu \mathrm{mol}$ between 0 and $12 \mathrm{~h}$ (observed $2.3 \mu \mathrm{mol}$ ), and $3.3 \mu \mathrm{mol}$ between 0 and $24 \mathrm{~h}$ (observed $3.6 \mu \mathrm{mol})^{[54]}$.

The liver plays an important role in the human body and is involved in most of the metabolism and elimination of drugs. On this basis, the system was designed and developed and may be more suitable for a drug whose metabolism mainly occurs in the liver, based on the current research. For drugs metabolized in the intestine or other organs, this system may also achieve simulation and prediction by replacing the corresponding microsomes entrapped in the FAB hydrogels, but this hypothesis still requires further validation. For drugs whose renal elimination and transporters play an important role in disposition or for macromolecular drugs with a limited diffusion rate, the current system may require further optimization and integration of additional mechanisms. In brief, the system tries to better model drug metabolism from the perspective of in vitro dynamics. However, the metabolic behavior of the drug is very complicated, and the system must be further refined and optimized, especially for drugs with a complicated metabolic mechanism.

In summary, a dynamic in vitro metabolic system based on peristaltic pumps and human liver microsomal encapsulated FAB hydrogel was constructed. The integration and application of microdialysis further improved the system. Compared to the traditional incubation method, more reasonable PK profile and in vivo clearance were predicted by the PBPK model integrated with the intrinsic clearance obtained from the BioPK system. The successful prediction maximized confidence in the metabolic reaction prediction obtained using the Bio-PK metabolic system, demonstrating the feasibility of applying the dynamic system to screen compounds and to understand the metabolic mechanisms, especially for metabolism involving human study. This novel dynamic system also has the potential to further develop in vitro dynamic drug-drug interaction studies. A reliable in vitro dynamic DDI model in the human is also highly desired. This research provides a basis and support for later development and research.

\section{Acknowledgements}

This study was supported by a grant from the National Natural Science Foundation of China (Grant No 81673501). The Bio-PK metabolic system presented in this report is the subject of a granted patent by Jun-fen XING and Bin ZHU in China ("In vitro analyte detection for method and system of biological effect," Ratification № ZL200610118351.8).

\section{Author contribution}

Cai-fu XUE, Wei-min CAI and Bin ZHU designed the research; Cai-fu XUE, Zhe ZHANG and Yan JIN performed the research and analyzed the data; Cai-fu XUE and Zhe ZHANG wrote the paper; Wei-min CAI, Guo MA, Xiao-qiang XIANG and Jun-fen XING reviewed and revised the paper.

\section{References}

1 Venkatesh S, Lipper RA. Role of the development scientist in compound lead selection and optimization. J Pharm Sci 2000; 89: 145-54.

2 Hosea NA, Jones HM. Predicting pharmacokinetic profiles using in silico derived parameters. Mol Pharm 2013; 10: 1207-15.

3 Darnell M, Ulvestad M, Ellis E, Weidolf L, Andersson TB. In vitro evaluation of major in vivo drug metabolic pathways using primary human hepatocytes and HepaRG cells in suspension and a dynamic three-dimensional bioreactor system. J Pharmacol Exp Ther 2012; 343: 134-44.

4 Izadifar M, Baik OD, Alcorn J. Mechanistic modeling of drug elimination by the liver using local volume averaging method. Conf Proc IEEE Eng Med Biol Soc 2011; 2011: 4314-7.

5 Kang MJ, Song WH, Shim BH, Oh SY, Lee HY, Chung EY, et al. Pharmacologically active metabolites of currently marketed drugs: potential resources for new drug discovery and development. Yakugaku Zasshi 2010; 130: 1325-37.

6 Obach RS. Pharmacologically active drug metabolites: impact on drug discovery and pharmacotherapy. Pharmacol Rev 2013; 65: 578-640.

7 Smith DA, Obach RS. Metabolites and safety: What are the concerns, and how should we address them? Chem Res Toxicol 2006; 19: 1570-9.

8 Brandon EF, Raap CD, Meijerman I, Beijnen JH, Schellens $\mathrm{JH}$. An update on in vitro test methods in human hepatic drug biotransformation research: pros and cons. Toxicol Appl Pharmacol 2003; 189: 233-46.

9 Jia L, Liu X. The conduct of drug metabolism studies considered good practice (II): in vitro experiments. Curr Drug Metab 2007; 8: 822-9.

10 Nguyen HQ, Lin J, Kimoto E, Callegari E, Tse S, Obach RS. Prediction of Losartan-active carboxylic acid metabolite exposure following losartan administration using static and physiologically based pharmacokinetic models. J Pharm Sci 2017; 106: 2758-70.

11 Zimmerman HJ. Acetaminophen hepatotoxicity. Clin Liver Dis 1998; (2): 523-41.

12 Lavé T, Coassolo P, Reigner B. Prediction of hepatic metabolic clearance based on interspecies allometric scaling techniques and in vitro-in vivo correlations. Clin Pharmacokinet 1999; 36: 211-31.

13 Prot JM, Videau O, Brochot C, Legallais C, Benech H, Leclerc E. A cocktail of metabolic probes demonstrates the relevance of primary human hepatocyte cultures in a microfluidic biochip for pharmaceutical drug screening. Int J Pharm 2011; 408: 67-75.

14 Sahi J, Grepper S, Smith C. Hepatocytes as a tool in drug metabolism, transport and safety evaluations in drug discovery. Curr Drug Discov 
Technol 2010; 7: 188-98.

15 Sengupta S, Johnson BP, Swanson SA, Stewart R, Bradfield CA, Thomson JA. Aggregate culture of human embryonic stem cell-derived hepatocytes in suspension are an improved in vitro model for drug metabolism and toxicity testing. Toxicol Sci 2014; 140: 236-45.

16 Jones HM, Houston JB. Substrate depletion approach for determining in vitro metabolic clearance: time dependencies in hepatocyte and microsomal incubations. Drug Metab Dispos 2004; 32: 973-82.

17 Klopf W, Worboys P. Scaling in vivo pharmacokinetics from in vitro metabolic stability data in drug discovery. Comb Chem High Throughput Screen 2010; 13: 159-69.

18 Obach RS. Predicting clearance in humans from in vitro data. Curr Top Med Chem 2011; 11: 334-9.

19 Lee J, Kim SH, Kim YC, Choi I, Sung JH. Fabrication and characterization of microfluidic liver-on-a-chip using microsomal enzymes. Enzyme Microb Technol 2013; 53: 159-64.

20 Yang $\mathrm{H}$, Zheng Y, Zhao B, Shao T, Shi Q, Zhou N, et al. Encapsulation of liver microsomes into a thermosensitive hydrogel for characterization of drug metabolism and toxicity. Biomaterials 2013; 34: 9770-8.

21 Zhou $\mathrm{N}$, Zheng $\mathrm{Y}$, Xing J, Yang $\mathrm{H}$, Chen $\mathrm{H}$, Xiang $\mathrm{X}$, et al. Application of a new dynamic model to predict the in vitro intrinsic clearance of tolbutamide using rat microsomes encapsulated in a Fab hydrogel. Drug Metab Dispos 2016; 44: 40-9.

22 Tong S, Sun H, Xue C, Chen H, Liu J, Yang H, et al. Establishment and assessment of a novel in vitro bio-PK/PD system in predicting the in vivo pharmacokinetics and pharmacodynamics of cyclophosphamide. Xenobiotica 2017; 6: 1-8.

23 Huang $\mathrm{H}$, Zhang $\mathrm{Y}$, Yang $\mathrm{R}$, Tang $\mathrm{X}$. Determination of baicalin in rat cerebrospinal fluid and blood using microdialysis coupled with ultraperformance liquid chromatography-tandem mass spectrometry. J Chromatogr B Analyt Technol Biomed Life Sci 2008; 874: 77-83.

24 Lanckmans K, Stragier B, Sarre S, Smolders I, Michotte Y. Nano-LCMS/MS for the monitoring of angiotensin IV in rat brain microdialysates: limitations and possibilities. J Sep Sci 2007; 30: 2217-24.

25 Qian M, West W, Wu JT, Lu B, Christ DD. Development of a dog microdialysis model for determining synovial fluid pharmacokinetics of anti-arthritis compounds exemplified by methotrexate. Pharm Res 2003; 20: 605-10.

26 Rodgers T, Rowland M. Physiologically based pharmacokinetic modelling 2: predicting the tissue distribution of acids, very weak bases, neutrals and zwitterions. J Pharm Sci 2006; 95: 1238-57.

27 De Buck SS, Sinha VK, Fenu LA, Nijsen MJ, Mackie CE, Gilissen RA. Prediction of human pharmacokinetics using physiologically based modeling: a retrospective analysis of 26 clinically tested drugs. Drug Metab Dispos 2007; 35: 1766-80.

28 Jones HM, Parrott N, Jorga K, Lavé T. A novel strategy for physiologically based predictions of human pharmacokinetics. Clin Pharmacokinet 2006; 45: 511-42.

29 Parrott N, Paquereau N, Coassolo P, Lave T. An evaluation of the utility of physiologically based models of pharmacokinetics in early drug discovery. J Pharm Sci 2005; 94: 2327-43.

30 Poulin P, Theil FP. Prediction of pharmacokinetics prior to in vivo studies. 1. Mechanism-based prediction of volume of distribution. J Pharm Sci 2002; 91: 129-56.

31 Yamazaki S, Skaptason J, Romero D, Vekich S, Jones HM, Tan W, et al. Prediction of oral pharmacokinetics of cMet kinase inhibitors in humans: physiologically based pharmacokinetic model versus traditional one compartment model. Drug Metab Dispos 2011; 39: 383-93.

32 Madsen H, Enggaard TP, Hansen LL, Klitgaard NA, Brøsen K. Fluvoxamine inhibits the CYP2C9 catalyzed biotransformation of tolbutamide. Clin Pharmacol Ther 2001; 69: 41-7.

33 Back DJ, Tjia J, Mönig H, Ohnhaus EE, Park BK. Selective inhibition of drug oxidation after simultaneous administration of two probe drugs, Antipyrine and Tolbutamide. Eur J Clin Pharmacol 1988; 34: 157-63.

34 Kanamitsu S, Ito K, Sugiyama Y. Quantitative prediction of in vivo drug-drug interactions from in vitro data based on physiological pharmacokinetics: use of maximum unbound concentration of inhibitor at the inlet to the liver. Pharm Res 2000; 17: 336-43.

35 Veronese ME, Miners JO, Randles D, Gregov D, Birkett DJ. Validation of the tolbutamide metabolic ratio for population screening with use of sulfaphenazole to produce model phenotypic poor metabolizers. Clin Pharmacol Ther 1990; 47: 403-11.

36 Wing LM, Miners JO. Cotrimoxazole as an inhibitor of oxidative drug metabolism: effects of trimethoprim and sulphamethoxazole separately and combined on tolbutamide disposition. $\mathrm{Br} J \mathrm{Clin}$ Pharmacol 1985; 20: 482-5.

37 Miners JO, Wing LM, Lillywhite KJ, Smith KJ. Failure of 'therapeutic' doses of beta-adrenoceptor antagonists to alter the disposition of tolbutamide and lignocaine. Br J Clin Pharmacol 1984; 18: 853-60.

38 Wilner KD, Gardner MJ. Tenidap sodium does not alter the clearance or plasma protein binding of tolbutamide in healthy male volunteers. Br J Clin Pharmacol 1995; 39: 39S-42S.

39 Hemeryck A, De Vriendt C, Belpaire FM. Inhibition of CYP2C9 by selective serotonin reuptake inhibitors: in vitro studies with tolbutamide and (S)-warfarin using human liver microsomes. Eur J Clin Pharmacol 1999; 54: 947-51.

40 Miners JO, Smith KJ, Robson RA, McManus ME, Veronese ME, Birkett DJ. Tolbutamide hydroxylation by human liver microsomes. Kinetic characterisation and relationship to other cytochrome P-450 dependent xenobiotic oxidations. Biochem Pharmacol 1988; 37: 1137-44.

41 Schary WL, Rowland M. Protein binding and hepatic clearance: studies with tolbutamide, a drug of low intrinsic clearance, in the isolated perfused rat liver preparation. J Pharmacokinet Biopharm 1983; 11: 225-43.

42 Worboys PD, Bradbury A, Houston JB. Kinetics of drug metabolism in rat liver slices:rates of oxidation of ethoxycoumarin and tolbutamide, examples of high- and low-clearance compounds. Drug Metab Dispos 1995; 23: 393-7.

43 Brunner M, Derendorf H, Müller M. Microdialysis for in vivo pharmacokinetic/pharmacodynamic characterization of anti-infective drugs. Curr Opin Pharmacol 2005; 5: 495-9.

44 Romanyshyn L, Tiller PR, Hop CE. Bioanalytical applications of 'fast chromatography' to high-throughput liquid chromatography/tandem mass spectrometric quantitation. Rapid Commun Mass Spectrom 2000; 14: 1662-8.

45 Davies MI, Cooper JD, Desmond SS, Lunte CE, Lunte SM. Analytical considerations for microdialysis sampling. Adv Drug Deliv Rev 2000; 45: 169-88.

46 Ungerstedt U. Microdialysis--principles and applications for studies in animals and man. J Intern Med 1991; 230: 365-73.

47 Xue C, Guo J, Qian D, Duan JA, Shang E, Shu Y, et al. Identification of the potential active components of Abelmoschus manihot in rat blood and kidney tissue by microdialysis combined with ultra-performance liquid chromatography/quadrupole time-of-flight mass spectrometry. J Chromatogr B Analyt Technol Biomed Life Sci 2011; 879: 317-25.

48 Guo P, Wang X, Liu L, Belinsky MG, Kruh GD, Gallo JM. Determination of methotrexate and its major metabolite 7-hydroxymethotrexate in mouse plasma and brain tissue by liquid chromatographytandem mass spectrometry. J Pharm Biomed Anal 2007; 43: 1789-95.

49 AO X, Stenken JA. Water-soluble cyclodextrin polymers for enhanced 
relative recovery of hydrophobic analytes during microdialysis sampling. Analyst 2003; 128: 1143-9.

50 Wang $\mathrm{H}$, Wang Z, Lu M, Zou $\mathrm{H}$. Microdialysis sampling method for evaluation of binding kinetics of small molecules to macromolecules. Ana Chem 2008; 80: 2993-9.

51 Semple HA, Tam YK, Coutts RT. A computer simulation of the food effect: transient changes in hepatic blood flow and MichaelisMenten parameters as mediators of hepatic first pass metabolism and bioavailability of propranolol. Biopharm Drug Dispos 1990; 11: 61-76.

52 Miners JO, Birkett DJ. Use of tolbutamide as a substrate probe for human hepatic cytochrome P450 2C9. Methods Enzymol 1996; 272: 139-45.

53 Barter ZE, Tucker GT, Rowland-Yeo K. Differences in cytochrome P450-mediated pharmacokinetics between chinese and caucasian populations predicted by mechanistic physiologically based pharmacokinetic modelling. Clin Pharmacokinet 2013; 52: 1085-100.

54 Chen K, Wang R, Wen SY, Li J, Wang SQ. Relationship of P450 2C9 genetic polymorphisms in Chinese and the pharmacokinetics of tolbutamide. J Clin Pharm Ther 2005; 30: 241-9.

55 Dickinson GL, Rezaee S, Proctor NJ, Lennard MS, Tucker GT, Rostami-Hodjegan A. Incorporating in vitro information on drug metabolism into clinical trial simulations to assess the effect of Cyp2d6 polymorphism on pharmacokinetics and pharmacodynamics: dextromethorphan as a model application. J Clin Pharmacol 2007; 47: $175-86$.

56 Tassaneeyakul W, Veronese ME, Birkett DJ, Doecke CJ, McManus ME, Sansom LN, et al. Co-regulation of phenytoin and tolbutamide metabolism in humans. Br J Clin Pharmacol 1992; 34: 494-8.

57 Ito K, Houston JB. Comparison of the use of liver models for predicting drug clearance using in vitro kinetic data from hepatic microsomes and isolated hepatocytes. Pharm Res 2004; 21: 785-92. 